[8] S. Marcus, Fonctions convexes et fonctions internes, Bull. Sci. Math. 81 (1957), pp. $66-70$.

[9] - Sur une classe de fonctions définies par des inégalités, introduite par ML. Á, Osászúr, Acta Sci. Math. (Szeged) 19 (1958), pp. 192-218.

[10] R. M. Solovay, A model of set-theory in which every set of reals is Lebesgue measurable, Ann. of Math. 92 (1970), pp. 1-56.

Accepté par la Rédaction le 16. 1. 1974

\section{Concerning unicoherence of continua}

by

Ivan Guintchev (Rousse)

Abstract. In this paper we investigate the unicoherence of a continuum $M$, knowing that the elements of a certain decomposition $\mathcal{S}$ of $M$ are unicoherent. We confine ourselves to considering only upper semi-continuous monotone decompositions. In this case, if the decomposition space $M / \mathcal{G}$ is a dendroid and for each subcontinuum $K$ of $M$ each element $G$ of $\mathcal{S}$ the intersection $K \cap G$ is a continuum, then $M$ is unicoherent.

If $M$ is a hereditarily decomposable continuum which is irreducible about a finite set and $\mathcal{S}$ is an admissible decomposition of $M$, the suppositions may reduce to the single one that the elements of $\mathcal{G}$ are unicoherent.

We obtain analogous assertions concerning the hereditary unicoherence of continua if the elements of $\mathcal{G}$ are such.

In this paper a continuum means a compact connected metric space.

Let $M$ be a continuum. A family $\mathbf{S}$ of closed disjoint subsets of $M$ covering $M I$ is said to be a decomposition of $M$.

The decomposition $\mathbf{S}$ of $M$ is said to be monotone if its elements are continua.

The decomposition $\mathbf{G}$ of $M$ is said to be upper semi-continuous if for each open subset $U$ of $M$ containing some element $G$ of $\mathcal{G}$ there exists an open subset $V$ of $I I$ such that $G \subset V \subset U$ and $V$ is the union of the elements of $\mathcal{G}$ intersecting it. For equivalent definitions of this concept see [3], pp. 183-185, or [8], p. 122.

Let $I$ be a continuum irreducible from $a$ to $b$. Suppose that one can define a non-trivial upper semi-continuous monotone decomposition $\mathbb{G}$ of $I$ such that each element of $\mathbf{S}$ not containing $a$ and $b$ separates $I$. It is shown in [7] that in this case there exists a unique decomposition which is minimal with respect to the above properties. Its elements are called layers of $I$.

The upper semi-continuous monotone decomposition $\mathbf{G}$ of the continuum $M$ is said to be admissible if, for each irreducible continuum $I \subset M$ and for each layer $T$ of $I$, there exists an element $G$ of $\mathcal{G}$ containing $T$ (compare [2], p. 115).

A dendroid means an arcwise connected and hereditarily unicoherent continuum. 
A $\lambda$-dendroid means a hereditarily decomposable and hereditarily unicoherent continuum.

Let $M$ be a hereditarily decomposable continuum, which is irreducible about a finite set $W$ and let $w \in W$. It is shown in [6] that tho set $E=\{x \in M: M$ is irreducible about $\{x\} \cup(W \backslash\{w\})\}$ is a continuum. We call $D$ an end-continunm of $M$. This result of [6] generalizes an analogous result for irreducible continua obtained in [5]. In this paper we purpose to show that a continuum. $M$ is unicoherent or hereditarily unicoherent if the elements of a certain decomposition of $M T$ possess the same property. In particular, we consider the case where $M$ is hereditarily decomposable and irreducible about the finite set.

THeorem 1. If an upper semi-continuous monotone decomposition of of the continum $M$ is such that

$1^{0}$ the elements of $\mathcal{G}$ are unicoherent,

$2^{\circ}$ the decomposition space $\mathbb{M} / \mathcal{S}$ is a dendroid,

$3^{\circ}$ for each subcontinuum $K$ of $M$ and each element $G$ of $S$ the intersection $K \cap G$ is a continuum,

then $M$ is unicoherent.

Proof. Denote the decomposition space $M / \mathcal{G}$ by $T$ and the quotient map of $M$ onto $T$ by $f$. Thus $f: M \rightarrow M / \mathcal{G}=T$ is monotone.

Let $M=K \cup L$, where' $K$ and $L$ are proper subcontinua of $M$. The set $K \cup L$ is closed. We shall prove that it is strongly connected.

Let $a$ and $b$ be arbitrary points of $K \cap L$ and put $a^{\prime}=f(a)$ and $b^{\prime}=f(b)$. By $2^{\circ}$ the space $T$ is a dendroid, so there exists a unique aro $\left(a^{\prime}, b^{\prime}\right) T$ having $a^{\prime}$ and $b^{\prime}$ as its end-points. Let $S=f^{-1}\left(\left(a^{\prime}, b^{\prime}\right)\right)$. Since $f$ is monotone, $S$ is a continuum.

Let $K_{1}=K \cap S$ and $L_{1}=L \cap S$. We shall prove that $K_{1}$ and $L_{1}$ are continua.

Let $K^{*}$ denote a subcontinuum of $K$ which is irreducible from $a$ to $b$. Put $f_{1}=\left.f\right|_{K^{*}}$. If $t \in f_{1}\left(K^{*}\right)$, then $f_{1}^{-1}(t)=K^{*} \cap f^{-1}(t)$. The set $f^{-1}(t)$ is an element of $\mathcal{S}$ and from $3^{\circ}$ we see that $f_{1}^{-1}(t)$ is a continuum. Thus the map $f_{1}$ is monotone. Since $K^{*}$ is irreducible from $a$ to $b$, and since $f_{1}$ is monotone, $f\left(K^{*}\right)=f_{1}\left(K^{*}\right)$ is irreducible from $a^{\prime}=f_{1}(a)=f(a)$ to $b^{\prime}=f_{1}(b)=f(b)$ (see [4], § 48, Theorem 3, p. 192). Thus $f\left(K^{*}\right) \subset T$ is irreducible from $a^{\prime}$ to $b^{\prime}$, and since $T$ is a dendroid, there exists a unique subcontinum of $T$ irreducible from $a^{\prime}$ to $b^{\prime}$, namely the arc $\left(a^{\prime}, b^{\prime}\right)$. Thus $f\left(K^{*}\right)=\left(a^{\prime}, b^{\prime}\right)$ and therefore $K^{*} \subset f^{-1}\left(f\left(K^{*}\right)\right)=f^{-1}\left(\left(a^{\prime}, b^{\prime}\right)\right)=\$$.

Suppose $K_{1}=K \cap S=A \cap B$, where $A$ and $B$ are closed disjoint sets and $A \neq \varnothing \neq B$. Let $a \in A$. The continuum $K^{*}$ is contained in both $S$ and $K$ and therefore $K^{*}$ is contained in $K \frown S$. Since $K^{*}$ is connected and $K^{*}$ contains both $a$ and $b$, we have $K \subset A$ and $b \in A$.
Let $c \in B$ and let $G_{c}$ be the element of $\mathcal{G}$ which contains $c$, i.e., $c \in G_{c} \in \mathcal{G}$. Since $G_{c} \cap S$ contains $c$, we have $G_{c} \subset S$, whence $K \cap G_{c} \subset K \cap S$. By $3^{\circ}$ the set $K \cap G_{c}$ is connected and since $c$ belongs to both $B$ and $K \cap G_{c}$, we have $K \cap G_{c} \subset B$. The points $a$ and $b$ on the one hand and $c$ on the other hand belong to different components of $K_{1}$; therefore $a \neq c \neq b$. Moreover, since for each $G \in \mathcal{G}$ the set $K \cap G$ is connected by $3^{\circ}, K \cap G_{a}$ and $K \cap G_{b}$ on the one hand and $I \cap G_{c}$ on the other are contained in different components of $K_{1}$, and therefore $G_{a} \neq G_{c} \neq G_{b}$ (here $G_{a}$ and $G_{b}$ denote the elements of $\mathcal{S}$ containing $a$ and $b$, respectively).

Observe that the set $S \backslash G_{c}$ is not connected by the construction, and that points $a$ and $b$ are in different components of $S \backslash G_{c}$. Since both $a$ and $b$ belong to $K$, we see that $K \cap\left(S \backslash G_{c}\right)$ is not connected and it contains $a$ and $b$ in different components of it. Since $K^{*} \subset A, K \cap G_{c} \subset B$ and $A \cap B=\varnothing$, we have $K^{*} \cap\left(K \cap G_{c}\right)=\varnothing$, and we conclude from $K^{*} \subset K \cap S$ that $K^{*} \subset K \cap S \backslash\left(K \cap G_{c}\right)=K \cap\left(S \backslash G_{c}\right)$. Therefore $a$ and $b$, as. points of the continuum $K^{*}$, belong to the same component of $K \cap\left(S \backslash G_{c}\right)$ : a contradiction. This proves that $K_{1}$ (and similarly $L_{1}$ ) is connected. Since they are closed by definition, we have just proved that $K_{1}$ and $L_{1}$ are continua.

Let us consider sets of the type $G \cap K_{1} \cap L_{1}$, where $G$ is an element. of $\mathcal{G}$. For each $G \in \mathcal{S}$ such that $G \subset S$ the following properties hold.

$1^{\circ}$ The intersections $G \cap K_{1}$ and $G \cap L_{1}$ are both non-empty.

In fact, suppose that $G \cap K_{1}=\varnothing$ for some $G$. Thus

(1)

$$
K_{1}=K_{1} \backslash G \cap K_{1}=K \cap S \backslash G \cap K \cap S=K \cap(S \backslash G) .
$$

Since $a, b \in K_{1}$, we see by the assumption that $a$ and $b$ are not in $G$; therefore they are in different components of the set $S \backslash G$, and thus of the intersection $K \cap(S \backslash G)$; a contradiction of (1).

$2^{\circ}$ The intersection $G \cap K_{1} \cap L_{1}$ is non-empty.

In fact, by property $1^{\circ}$ we have $G \cap K_{1} \neq \varnothing$ and $G \cap L_{1} \neq \varnothing$.

$$
\begin{aligned}
\left(G \cap K_{1}\right) \cup\left(G \cap L_{1}\right) & =G \cap\left(K_{1} \cup L_{1}\right)=G \cap(K \cap S \cup L \cap S) \\
& =G \cap(S \cap(K \cup L))=G \cap S \cap M=G .
\end{aligned}
$$

Thus $G$ is represented as the union of two non-empty closed sets, namely $G \cap K_{1}$ and $G \cap L_{1}$. Since $G$ is connected, we have

$$
\left(G \cap K_{1}\right) \cap\left(G \cap L_{1}\right) \neq 0, \quad \text { i.e. } \quad G \cap K_{1} \cap L_{1} \neq \varnothing .
$$

$3^{\circ}$ The set $G \cap K_{1} \cap L_{1}$ is a continuum.

In fact, by $3^{\circ}$ we infer that $G \cap K_{1}$ and $G \cap L_{1}$ are continua, since $K_{1}$ and $L_{1}$ are. Formula (2) shows that $\left(G \cap K_{1}\right) \cup\left(G \cap L_{1}\right)=G$. Since $G$ is unicoherent by $1^{\circ}$, we conclude that $\left(G \cap K_{1}\right) \frown\left(G \cap L_{1}\right)=G \frown K_{1} \cap L_{1}$ is a continuum. 
We shall prove that $Q=K_{1} \cap L_{1}$ is a continuum. To prove this it is sufficient to show that $Q$ possesses only one component. Let us recall that the intersection of all open and closed (relatively to $Q$ ) subsets of $Q$ which contain a point $p \in Q$ is called a quasi-component of $Q$ containing $p$. Tf the set $Q$ is compact, the concept of a component of $Q$ and of a quasi-component of $Q$ coincide (see [4], $\S 47$, Theorem 2, p. 169). Further, if $Q$ is separable, there exists a continuous map $h: Q \rightarrow \mathcal{C}$ (here $\mathrm{C}$ is the well-known Cantor discontinuum) such that point inverses under $h$ are quasi-components of $Q$ (see [4], $\S 46$, Theorem 3, p. 148). Let us consider the map $f_{2}: Q \rightarrow\left(a^{\prime}, b^{\prime}\right)$ defined as the restriction of $f$ on. $Q$. Let $t$ be an arbitrary point of $\left(a^{\prime}, b^{\prime}\right)$ and let $G_{t}=f^{-1}(t) \in \mathcal{G}$. It follows by property $2^{\circ}$ that $G_{l} \cap$ $\cap K_{1} \cap L_{1} \neq \varnothing$, i.e., $G_{t} \cap Q \neq \varnothing$. The latter implies that $f_{2}\left(G_{t} \cap Q\right)=$ $=f\left(G_{t}\right)=\{t\}$. Thus $f_{2}$ maps $Q$ onto $\left(a^{\prime}, b^{\prime}\right)$. Observe further that according to properties $2^{\circ}$ and $3^{\circ} f_{2}^{-1}(t)=f^{-1}(t) \cap Q=G_{t} \cap K_{1} \cap L_{1}$ is a non-empty continuum. Thus $f_{2}^{-1}(t)$ is contained in only one component of $Q$ and since the set $f_{2}^{-1}(t)$ is non-empty; therefore putting $g(t)=h\left(f_{2}^{-1}(t)\right)$ we see that $g$ : $\left(a^{\prime}, b^{\prime}\right) \rightarrow \mathcal{C}$ is a well-defined map of $\left(a^{\prime} b^{\prime}\right)$ into $\mathrm{C}$. The map $g$ is defined in such a way that the following diagram commutes:

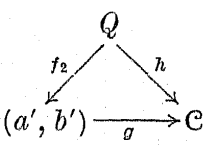

The map $f_{2}$ is continuous as a restriction of a continuous map. Let $F$ be an arbitrary closed subset of $C$. Since $h$ is continuous, $h^{-1}\left(F^{\prime}\right)$ is closed in $Q$, and since $Q$ is compact, $f_{2}\left(h^{-1}\left(F^{\prime}\right)\right)$ is closed. Thus the map $g$ is continuous. Therefore, $\left(a^{\prime}, b^{\prime}\right)$ being connected, its image $g\left(\left(a^{\prime}, b^{\prime}\right)\right)$ is a connected subset of $\mathrm{C}$, and since $\mathrm{C}$ is totally disconnected, we conclude that $g\left(\left(a^{\prime}, b^{\prime}\right)\right)$ is a set containing only one point. Further, $h(Q)=g\left(f_{2}(Q)\right)=g\left(\left(a^{\prime}, b^{\prime}\right)\right)$; therefore $Q=K_{1} \cap L_{1}$ has only one component. Thus the set $K_{1} \cap L_{1}$ is connected. Since it is obviously closed, it is a continuum.

The points $a$ and $b$ belong to both $K_{1}$ and $L_{1}$, and so they belong to the intersection $K_{1} \cap L_{1}$. Since $K_{1} \subset K$ and $L_{1} \subset L$, we have $K_{1} \cap L_{1} \subset$ $C K \cap L$. Thus the set $K \cap L$ is strongly connected, which completes the proof.

THEOREM 2. If an upper semi-continuous monotone decomposition s of the continuum $I$ L has properties $2^{\circ}$ and $3^{\circ}$ of Theorem 1, and 1.' the elements of $\mathcal{S}$ are hereditarity unicoherent, then $I M$ is hereditarily wnicoluerent.

Pro of. Let $P$ be an arbitrary subcontinum of $M$. Wo consider a decomposition $D$ of $P$ defined as follows:

$$
D=\{D \subset P: D=G \cap P \text { for some } G \in \mathscr{G}\} \text {. }
$$

The decomposition $D$ is monotone by $3^{\circ}$. It is upper semi-continuous, since $\mathcal{G}$ is upper semi-continuous and $P$ is a continuum (see [1], Lemma 1 , p. 117).

We shall prove that the decomposition $D$ has properties $1^{\circ}, 2^{\circ}$ and $3^{\circ}$ of Theorem 1.

Since each element of $\mathcal{G}$ is a subcontinuum of an element of $\mathcal{S}$, property $1^{\circ}$ follows by $1^{\prime}$.

To prove $2^{\circ}$, let us consider the decomposition space $P / D$ and let $f: M \rightarrow M / \mathcal{S}$ and $g: P \rightarrow P / D$ be the quotient maps. We shall define a map $h: P / D \rightarrow M / \mathcal{S}$. Let $t \in P / D$ be an arbitrary point and let $G_{t} \in \mathbf{S}$ be such that $g\left(G_{t} \cap P\right)=t$. The set $G_{t}$ is uniquely determined. We define $h(t)=$ $=f\left(G_{t}\right)$. The map $h$ is defined in such a way that the following diagram commutes:

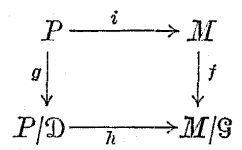

where $i$ denotes the inclusion mapping.

The map $h_{t}$ is injective. In fact, if $t_{1} \neq t_{2}, t_{j} \in P / D$, then $G_{t_{1}} \neq G_{t_{2}}$ and therefore $h\left(t_{1}\right) \neq h\left(t_{2}\right)$

Let $F$ be an arbitrary closed set of $M / \mathcal{S}$. Since $f$ is continuous, $f^{-1}\left(F^{\prime}\right)$ is a closed set in $M$; thus $P \cap f^{-1}(F)$ is closed in $P$ and since $P$ is a continuum and $g$ is continuous, the set $g\left(P \cap f^{-1}(F)\right)$ is closed in $P / D$. Thus the map $h$ is continuous, and since it is injective, $h$ is an imbedding of the continuum $P / D$ into the dendroid $M / \mathscr{S}$. Therefore $P / D$ is a dendroid.

To prove $3^{\circ}$, let $Q$ be an arbitrary subcontinuum of $P$ and let $D$ be an element of $D$. The set $Q \cap D=Q \cap G \cap P=Q \cap G$ is a continuum by $3^{\circ}$, which is assumed for the decomposition $\mathcal{G}$ of $M$.

Thus we have just shown that the hypotheses of Theorem 1 are satisfied for the decomposition $D$ of $P$. Hence $P$ is unicoherent and consequently $M$ is hereditarily unicoherent.

THEOREM 3. Let 9 be an admissible decomposition of the hereditarity decomposable continuum $M$. Then $M$ is a $\lambda$-dendroid if and only if properties $1^{\prime}, 2^{\circ}$ and $3^{\circ}$ assumed for $\mathcal{G}$ are satisfied.

Pro of. The sufficiency follows from Theorem 2. The necessity of properties $1^{\prime}$ and $3^{\circ}$ follows at once by the hereditary unicoherence of the $\lambda$-dendroids. The necessity of $2^{\circ}$ is established in [1], Theorem 5, p. 26.

LEMMA 1. Let $M$ be a continum which is irreducible about a finite set containing $n$ points and let $\mathbf{S}$ be an admissible decomposition of $M$. Then $M I / \mathcal{G}$ is a dendroid with at most $n$ ends.

Pro of. Let $M$ be irreducible about the set $\left\{a_{1}, a_{2}, \ldots, a_{n}\right\}$, let $f: M I \rightarrow \mathbb{M} / \mathbf{G}$ be the quotient map and let $a_{k_{2}}^{\prime}=f\left(a_{k}\right), 1 \leqslant k_{k} \leqslant n$. It is easy to verify 
that since $f$ is monotone, the continuum $M / \mathcal{S}$ is irreducible about the set $\left\{a_{1}^{\prime}, a_{2}^{\prime}, \ldots, a_{n}^{\prime}\right\}$ (the proof is exactly the same as in the case $n=2$ given in [4], $\S 48$, Theorem 3, p. 192).

In [2] (see [2], Theorem. 1, p. 116) it is shown that the decomposition space of an admissible decomposition is arcwise connected (even hereditarily arcwise connected). We denote by $l_{k}, 1 \leqslant k \leqslant n-1$, a fixed are of $M / S / S$ such that both $a_{l c}^{\prime}$ and $a_{n}^{\prime}$ belong to $l_{l}$. We define inductively continua $L_{k}, 1 \leqslant l_{k} \leqslant n-1$. Let $L_{1}=l_{1}$ and let $\alpha_{k} \in l_{k+1+1} \cap L_{k}$ be such that if:

$$
\left(\beta_{k}, \alpha_{k+1}^{\prime}\right) \mp\left(\alpha_{k}, a_{k+1}^{\prime}\right) \subset l_{k_{k}},
$$

then $\beta_{k} \notin L_{k}$. We define $L_{k+1}=L_{k} \cup\left(a_{k}, a_{k+1}^{\prime}\right)$, where $\left(a_{k}, a_{k+1}^{\prime}\right) \subset l_{k+1}$. For each $k, 1 \leqslant k \leqslant n-1$, the set $I_{K}$ is a dendroid with at most $k+1$. ends by construction.

Since $L_{n-1}$ contains each point $a_{k}^{\prime}, 1 \leqslant k \leqslant n$, and since $M / \mathcal{G}$ is irreducible about the set $\left\{a_{1}^{\prime}, a_{2}^{\prime}, \ldots, a_{n}^{\prime}\right\}$, we see that $L_{n-1}=M / \mathcal{S}$, which finishes the proof.

LemMa 2. Let $M$ be a hereditarily decomposable continuum which is irredncible about a finite set containing $n$ points and let $\mathbf{S}$ be an upper semicontinuous monotone decomposition of $M$ such that the decomposition space $M . \mathcal{G}$ is a dendroid. If $G$ is an element of $\mathfrak{S}$, then

(a) $M \backslash G=U_{1} \cup U_{2} \cup \ldots \cup U_{k}$, where $k \leqslant n$ and where $U_{i}, 1 \leqslant i \leqslant k$, are open connected sets.

(b) For each $i, 1 \leqslant i \leqslant k$, the set $U_{i}$ is irreducible about a finite set and it has $\bar{U}_{i} \cap G$ as its end-continum.

Proof. (a) Among the components of $M \backslash G$ we choose $U_{1}, U_{2}, \ldots, U_{k}$, where $k \leqslant n$, such that every end-continuum of $M$ which is not contained in $G$ intersects some $U_{i}, 1 \leqslant i \leqslant k$.

For each $i, 1 \leqslant i \leqslant k$, the union $U_{i} \cup G$ is a continuum. Indeed, either $U_{i} \cup G=M$ (in this case $k=1$ ) or $G$ separates $M$ into at least two different components. In the second case the above statement is established in [4], $\$ 47$, as Theorem 3 on p. 168

It follows that $\bigcup_{i=1}^{k} U_{i} \cup G$ is a continum and since it intersects all the end-continua of $M$, we have $M=\bigcup_{i=1}^{k} U_{i} \cup G$. Therefore $M \backslash Q=\bigcup_{i=1}^{k} U_{i}$, where $k$ is at most $n$. Obviously we have

$$
U_{i}=M \backslash\left\{U_{j} \cup G: j=1,2, \ldots, i-1, i+1, \ldots, k\right\} .
$$

and since $\cup\left\{U_{i} \cup G: j=1,2, \ldots, i-1, i+1, \ldots, k\right\}$ is a continuum; therefore $U_{i}$ is an open set. (b) Let $i$ denote a fixed natural number, $1 \leqslant i \leqslant k$, let $U_{i}$ intersect $s$ end-continua of $M: E_{1}, E_{2}, \ldots, E_{s}$, let $a_{j} \in E_{j} \cap D_{i}, 1 \leqslant j \leqslant s$, and let $x \in \bar{U}_{i} \cap G$.

We denote by $M^{\prime}$ a continuum which is irreducible about the set $\left\{x, a_{1}, \ldots, a_{s}\right\}$ and which is contained in $\bar{U}_{i}$. Observe that $M I^{\prime} \cup\left(M \backslash U_{i}\right)=M$, since $M M^{\prime} \cup\left(M \backslash U_{i}\right)$ is a continuum intersecting all the ends of $M$. Thus $\bar{U}_{i} \subset M^{\prime}$, whence $\bar{U}_{i} \subset M M^{\prime}$, and consequently we have $M^{\prime}=\bar{U}_{i}$. Therefore $\bar{U}_{i}$ is irreducible about $s+1$. points and the set $\bar{U}_{i} \cap G$ is contained in one of the end-continua of $U_{i}$.

We shall prove that if $x_{1}$ does not belong to $\bar{U}_{i} \cap G$, there exists a proper subcontinuum $A$ of $\bar{U}_{i}$ such that $A$ contains $x_{1}$ and all the points $a_{j}$, $1 \leqslant j \leqslant s$. Since $\boldsymbol{G}$ is monotone, the quotient map $f: M \rightarrow M / \mathcal{G}$ is monotone. We put $a_{j}^{\prime}=f\left(a_{j}\right), 1 \leqslant j \leqslant s$. Let $x_{1} \in D_{i}$ and let $x_{1}^{\prime}=f\left(x_{1}\right)$. Since $O_{i}$ is connected, $f\left(U_{i}\right)$ is connected, and since $a_{j}^{\prime} \in f\left(U_{j}\right)$ and $M / \mathcal{G}$ is a dendroid, we conclude that $\left(a_{j}^{\prime}, x_{1}^{\prime}\right) \subset f\left(U_{i}\right)$ and consequently $A^{\prime}=\bigcup_{j=1}^{s}\left(a_{j}^{\prime}, x_{1}\right) \subset f\left(U_{i}\right)$. The set $A^{\prime}$ is a continuum as a finite union of arcs which have the point $x_{1}^{\prime}$ in common. Since the map $f$ is monotone, $A=f^{-1}\left(A^{\prime}\right)$ is a continuum contained in $U_{i}$ and containing the set $\left\{x_{1}, a_{1}, \ldots, a_{s}\right\}$. Therefore $x_{1}$ does not belong to the end-continuum of $\bar{U}_{i}$ containing $\bar{U}_{i} \cap G$, which shows that the set $\bar{U}_{i} \cap G$ is an end-continuum of $\mathbb{M}$. The proof of Lemma 2 is finished.

LEMMA 3. Let $M$ be a hereditarity decomposable continuum which is irreducible about a finite set and let $\mathrm{G}$ be an upper semi-continuous monotone decomposition of $M$ such that the decomposition space $M / G$ is a dendroid. If $K$ is a subcontinuum of $M$, then for each element $G$ of $\mathcal{G}$ the intersection $K \cap G$ is a continuum.

Proof. Suppose that for some subcontinuum $K$ of $M$ and for some $G \in \mathcal{G}$ the intersection $I \cap G$ is not a continuum, i.e., $K \cap G=X \cup I$ where $X$ and $Y$ are closed disjoint non-empty sets. By Lemma 2 we have $\not N \backslash G=U_{1} \cup U_{2} \cup \ldots \cup U_{k}, K \leqslant n$, where $U_{i}, 1 \leqslant i \leqslant k$, are open sets (here $n$ is the cardinality of the minimal set $W$ such that $M$ is irreducible about $W$ ). It is clear that $K$ intersects some of the sets $U_{i}$; otherwise $K \subset G$ and $K \frown G=K$ would be a continum.

We shall prove that if $K \cap U_{i} \neq \varnothing$, then $\bar{U}_{i} \cap G$ is contained in $K$. Let $x_{0} \in K \cap U_{i}$ and let $x_{2} \in K \cap G$. We choose continua $I_{1} \subset K$ irreducible from $x_{0}$ to $x_{1}$ and $I_{2} \subset U_{i}$ irreducible about $x_{0}$ and the end-continua of $M$ intersecting $U_{i}$. The existence of the latter is established by Lemma 2

The set $I_{1} \cup I_{2}$ is a continuum which intersects $M \backslash U_{i}$. Therefore $I_{1} \cup I_{2} \cup\left(M \backslash U_{i}\right)$ is a continuum intersecting all the end-continua of $M I$, which ensures that $I_{1} \cup I_{2} \cup\left(I \Lambda U_{i}\right)=U$. Thus $I_{1} \cup I_{2} \supset \bar{U}_{i}$. Therefore $\bar{U}_{i} \cap G \subset I_{1} \cup I_{2}$ and since $I_{2} \cap G=\emptyset$, we conclude that $\bar{U}_{i} \cap G \subset I_{1}$ i.e., $\bar{U}_{i} \cap G \subset K$ since $I_{1} \subset K$. 
We consider all the sets $U_{i}$ such that $U_{i} \cap K \neq \varnothing$. By the inclusior $\bar{U}_{i} \cap G \subset X \cup Y$ and since $\bar{U}_{i} \cap G$ is a continuum by Lemma 2, we obtail either $\bar{U}_{i} \cap G \subset X$ or $\bar{U}_{i} \cap G \subset X$.

Let $\left\{U_{p_{1}}, \ldots, U_{p_{0}}\right\}$ be all the sets among $U_{i}, 1 \leqslant i \leqslant k$, such that $K \cap U_{p_{j}} \neq \varnothing, 1 \leqslant j \leqslant s_{0}$, and $\bar{U}_{p_{j}} \cap G \subset X$

Let $\left\{U_{q_{1}}, \ldots, U_{q_{s 0}}\right\}$ be all the sets among $U_{i}, 1 \leqslant i \leqslant k$, such that $K \cap U_{q_{j}} \neq \varnothing, 1 \leqslant j \leqslant s_{1}$, and $\bar{U}_{q_{j}} \cap G \subset Y$.

$$
\begin{gathered}
K=K \cap M=K \cap\left(\bigcup_{i=1}^{k} U_{i} \cup G\right)=K \cap G \cup \bigcup_{j=1}^{s_{0}}\left(K \cap U_{p_{j}}\right) \cup \bigcup_{j=1}^{s_{1}}\left(K \cap U_{q_{j}}\right) \\
=K \cap G \cup \bigcup_{j=1}^{s_{0}}\left(K \cap \bar{U}_{p_{j}} \cap G\right) \cap \bigcup_{j=1}^{s_{1}}\left(K \cap \bar{U}_{\alpha_{j}} \cap G\right) \cup \\
\left.\quad \cup \bigcup_{j=1}^{s_{0}}\left(K \cap U_{p_{j}}\right) \cup \bigcup_{j=1}^{s_{1}} K \cap U_{q_{j}}\right),
\end{gathered}
$$

since the sets $\bar{U}_{p_{j}} \cap G, 1 \leqslant j \leqslant s_{0}$, and $\bar{U}_{q_{j}} \cap G, 1 \leqslant j \leqslant s_{1}$, are contained in $K \cap G$. Further, since $\bar{U}_{i} \subset U_{i} \cup G, 1 \leqslant i \leqslant k$, we obtain:

$$
\begin{aligned}
K & =K \cap G \cup \bigcup_{j=1}^{s_{0}}\left(K \cap \bar{U}_{p_{j}}\right) \cup \bigcup_{j=1}^{s_{1}}\left(K \cap \bar{U}_{q_{j}}\right) \\
& =X \cup \bigcup_{j=1}^{s_{0}} K \cap \bar{U}_{p_{j}} \cup Y \cup \bigcup_{j=1}^{s_{1}} K \cap \bar{U}_{q_{j}}=A \cup B,
\end{aligned}
$$

where

$$
A=X \cup \bigcup_{j=1}^{s_{0}} K \cap \bar{U}_{p_{j}} \text { and } \quad B=X \cup \bigcup_{j=1}^{s_{1}} K \cap \bar{U}_{\alpha_{j}} .
$$

We see that $A$ and $B$ are closed. Further,

$$
\begin{aligned}
& A=X \cap \bigcup_{j=1}^{s_{0}} K \cap \bar{U}_{p_{j}}=X \cup \bigcup_{j=1}^{s_{0}} K \cap\left(\bar{U}_{p_{j}} \cap G \cup U_{p_{j}}\right)=X \cup \bigcup_{j=1}^{s_{0}} K \cap U_{p_{j}}, \\
& B=Y \cup \bigcup_{j=1}^{s_{1}} K \cap \bar{U}_{q_{j}}=X \cup \bigcup_{j=1}^{s_{0}} K \cap\left(\bar{U}_{q_{j}} \cap G \cup U_{q_{j}}\right)=X \cup \bigcup_{j=1}^{s_{0}} K \cap U_{q_{j}} .
\end{aligned}
$$

Since $X \subset G$ and $X \subset G$, we have $U_{i} \cap K=\varnothing$ and $U_{i} \cap X=\emptyset$. Observe that $X \cap Y=\varnothing$, and $U_{i_{1}} \cap U_{i_{2}}=\varnothing$, where $i_{1} \neq i_{2}$. In aceordinee to these remarks we see that $A$ and $B$ are disjoint non-empty sets, which contradicts the assumption that $K$ is a continuum.

Lemmas 1 and 3 show that hereditarily decomposable continua which are irreducible about a finite set possess properties $2^{\circ}$ and $3^{\circ}$; hence we get the following theorems:

THEOREM 4. Let II be a hereditarily decomposable continuum which is irreducible about a finite set and let $\mathcal{G}$ be an admissible decomposition of $\mathbb{M}$. If the elements of $\mathcal{G}$ are unicoherent, so is $M$.
THEOREM 5. Let $M$ be a hereditarily decomposable continuum which is irreducible about a finite set and let $\mathbf{S}$ be an admissible decomposition of $\mathbb{M}$. Then $M$ is a $\lambda$-dendroid if and only if the elements of $\mathbb{G}$ are hereditarily. unicoherent.

Theorems 4 and 5 are direct consequences of Lemmas 1 and 3 and Theorems 1 and 3 .

We now discuss if all properties $1^{\prime}, 2^{\circ}$ and $3^{\circ}$ in Theorem 3 are essential for proving that $M$ is a $\lambda$-dendroid. In other words, we ask whether it is possible to omit one of these properties and still prove that $M$ is a $\lambda$-dendroid.

EXAMrPLE 1. If $M_{1}$ is a circle and if $\mathcal{G}$ is the point-decomposition, then both $1^{\prime}$ and $3^{\circ}$ hold, but $2^{\circ}$ does not.

EXAMPLE 2. If $M_{2}$ is the union of a circle and a ray approximating it and if $\mathcal{G}$ is an admissible decomposition of $M_{2}$, then both $2^{\circ}$ and $3^{\circ}$ hold, but $I^{\prime}$ does not.

Asking if property $3^{\circ}$ is essential, we come to an open question. Does a hereditarily decomposable continuum $M$ having properties $1^{\prime}$ and $2^{\circ}$ have also property $3^{\circ}$ \%

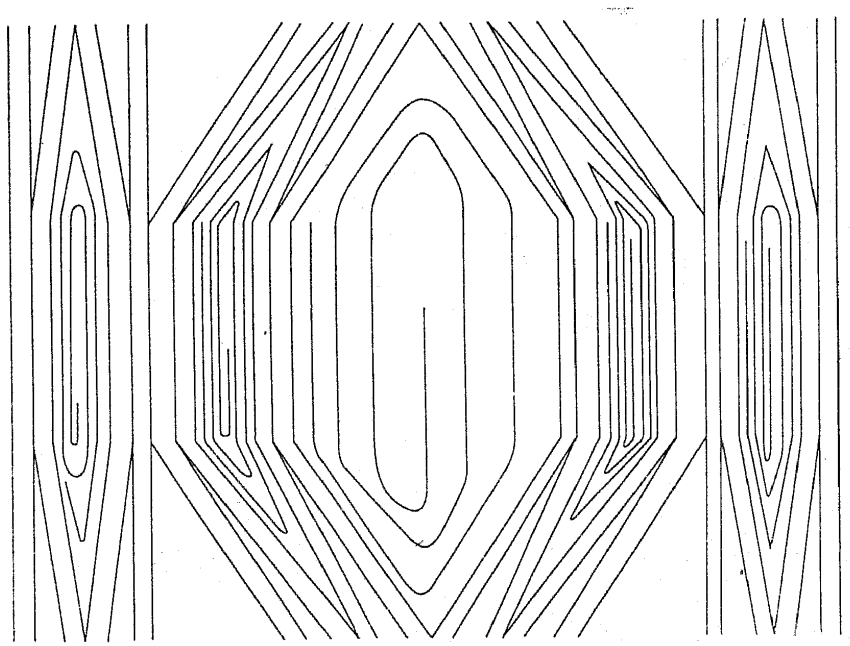

Fig. 1

Example 3 (this example is due to J. J. Charatonilk). If $M_{3}$ is the continuum shown in Fig. 1 and $\mathcal{G}$ is its minimal admissible decomposition, then $2^{\circ}$ is satisfied, but $3^{\circ}$ is not. In fact, $M_{3}$ is a continuum composed of 
two copies of the "accordion-like continuum" (see e.g. [7], p. 1.2), of uncountably many vertical straight line segments which join the corresponding layers of the copies, and of countably many spiral lines winding in to the simple closed curves contained in the continuum. To show the above proposition we just observe the subcontinuum of $M_{3}$ which is the union of both the "accordionlike continua" and an arbitrary segment connecting them.

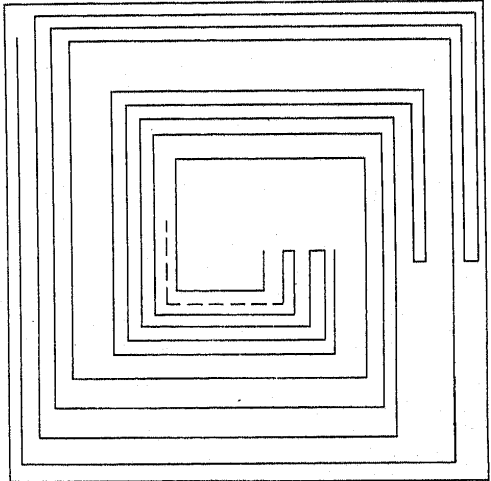

Fig. 2

EXAMPLE 4. We take the continuum $M_{3}$ and we approximate each simple closed curve which is an element of the minimal admissible decomposition of $M$ together with the spiral winding in to it by a rayy, as is shown in Fig. 2. We define the continuum $M_{4}$ as the union of $M_{3}$ and all the approximating rays. If $\mathcal{G}$ is the minimal admissible decomposition of $M_{4}$, then $1^{\circ}$ and $2^{\circ}$ hold, but $3^{\circ}$ does not

\section{References}

[1] J. J. Charatonik, On decompositions of $\lambda$-dendroids, Fund. Math. (67 (1970), pp. $15-30$.

[2] - On decompositions of continua, ibidem 79 (1973), pp. 113-130.

[3] K. Kuratowski, Topology, vol. I, Warszawa 1966.

[4] - Topology, vol. 2, Warszawa 1968.

[5] H. C. Miller, On unicoherent continua, Trans. Amer. Math. Soc. 69 (1950), pp. 174-194.

[6] M. J. Russoll, Monotone decompositions of continua irreducible about a finite set, Fund. Math. 72 (1971), pp. 255-264.
[7] E. S. Thomas, Jr., Monotone decompositions of irreducible continua, Rozprawy Ma. tematyczne 50, Warszawa 1966.

[8] G. T. Whyburn, Analytic Topology, Amer. Math. Soc. Coll. Publ. 38, New York 1942.

THE HIGHER INSTITUTE FOR MACHINE BUILDING, MECHANISATION

AND ELECTRIFICATION OF AGRICULTURE

Accepté par la Rédaction le 24. 1. 1974 